\title{
Closure of symptomatic coronary artery fistulae
}

\author{
Luis Antonio Íñigo-García ${ }^{1}$, Juan Luis Gutiérrez-Chico ${ }^{2}$, Teresa Gil-Jiménez ${ }^{1}$, \\ Luis Iñigo-Almansa ${ }^{3}$, Alberto Urbaneja-Salas ${ }^{3}$, Gabriel Rosas-Cervantes ${ }^{1}$ \\ ${ }^{1}$ Hospital Costa del Sol, Marbella, Spain \\ ${ }^{2}$ DRK-Klinikum Westend, Berlin, Germany \\ ${ }^{3}$ Clínica Santa Elena, Málaga, Spain
}

Presented herein are two cases of congenital coronary artery fistulae (CAF) treated with embolization coils, implanted by a joint team of interventional cardiologists and radiologists.

The first case is a 46-year-old woman, with chest pain after gastrointestinal surgery with elevated plasma levels of troponin, in whom a coronary angiography showed a coronary fistula of $1.8 \mathrm{~mm}$ from the proximal segment of the left anterior descending artery draining into the pulmonary artery. Percutaneous closure of the fistula was performed using three detachable coils produced by Boston Scientific (Fig. 1).

The second case is an 81-year-old woman admitted due to acute coronary syndrome and heart failure. The coronary angiography did not show significant coronary atherosclerosis but revealed a fistula of $2.2 \mathrm{~mm}$ in diameter, also from the proximal segment of the left anterior descending artery draining into the pulmonary artery. Percutaneous closure of the fistula was accomplished using two hydrogel coils produced by MicroVention, Terumo (Fig. 2).

During implantation differences were found between the devices, as the hydrogel coils closed the fistula more quickly, probably due to its hydrogel polymer coating that expands upon insertion. After 6 months follow-up both patients remain asymptomatic.

Coronary artery fistulae have low prevalence and their treatment options are not systematically detailed in current guidelines. Different therapies are currently available: surgical ligation, coils, microcoils, vascular plugs or covered stents.

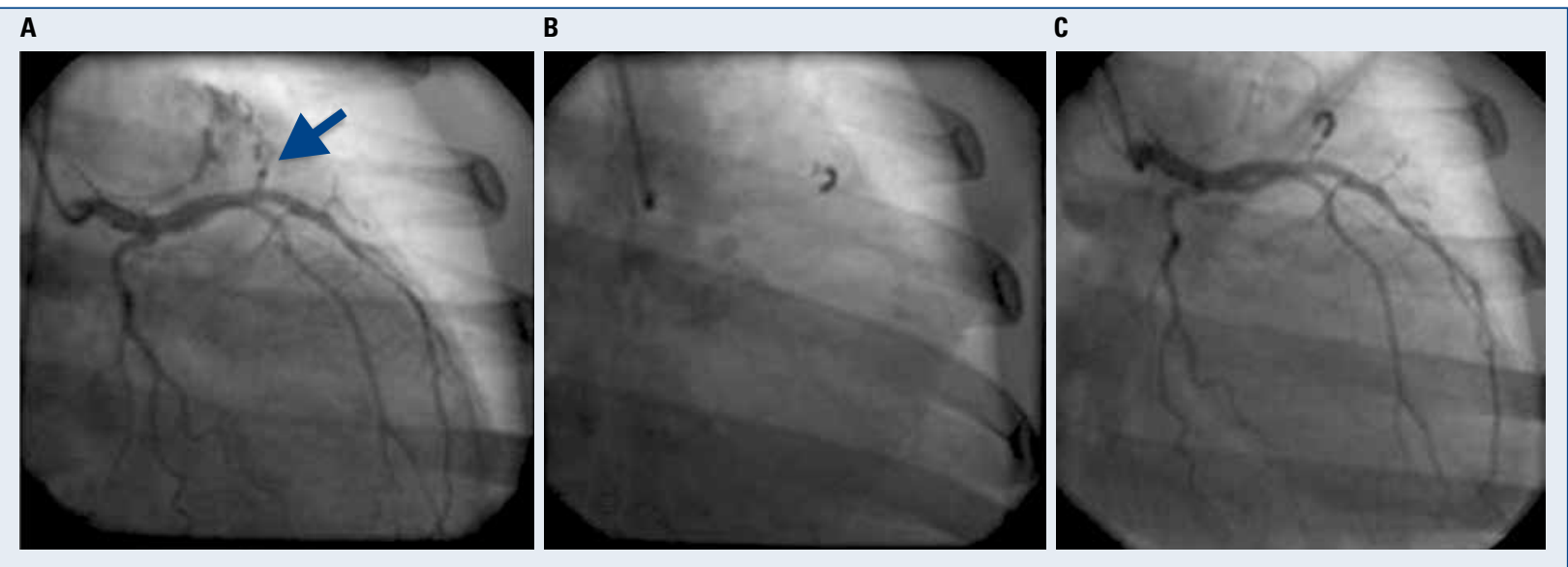

Figure 1. Angiographic image showing; A. Fistula connecting left anterior descending artery to pulmonary artery; B. Release of three detachable coils; C. Result after coil embolization.

Address for correspondence: Luis Antonio Ínigo-García, MD, Apartado de Correos, 22, 11310 - Puerto de Sotogrande, Spain, tel: 0034-667-923-743, e-mail: luis.igarcia@secardiologia.es

Received: 21.07.2017 Accepted: 25.07.2017 


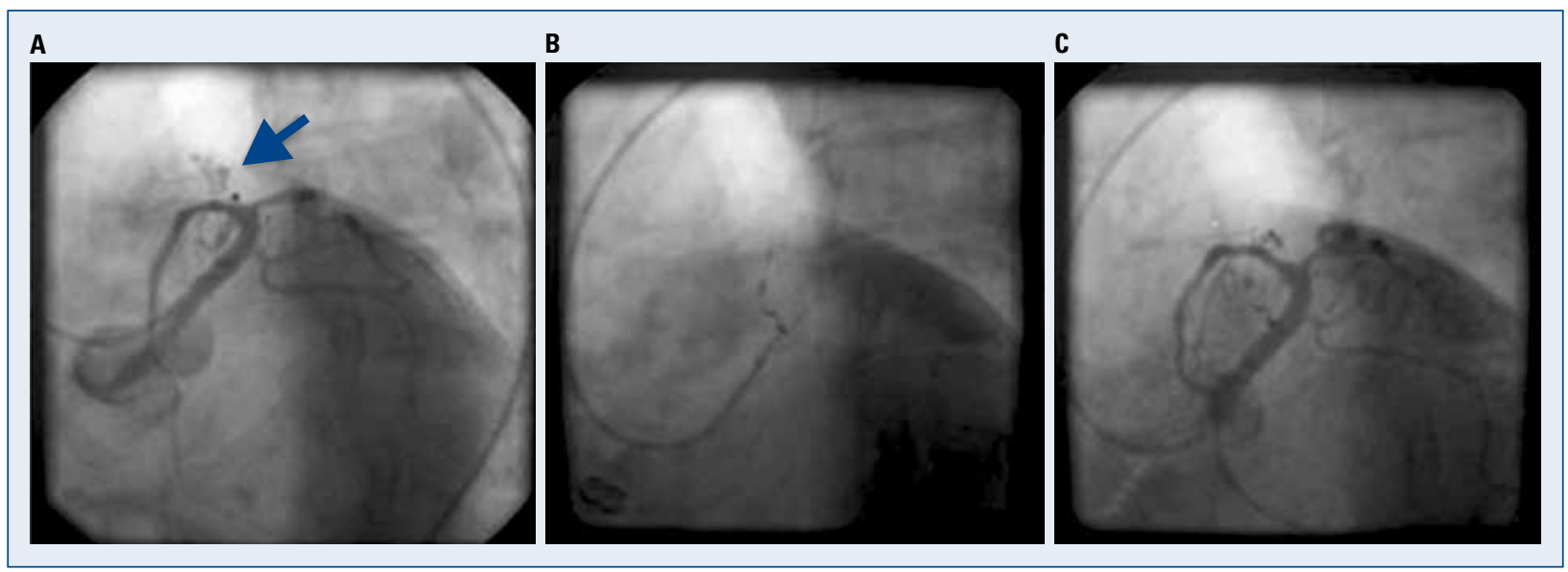

Figure 2. A. Fistula connecting left anterior descending artery to pulmonary artery; B. Release of two hydrogel coils; C. Coronary angiography showing complete occlusion of the fistula.

This study proposed the following therapeutic flow-chart: coils are the treatment of choice for small and medium-sized symptomatic fistulae, whilst vascular plugs should be the option for CAF of larger diameter. Surgical ligation should be lim- ited to cases of other concomitant cardiac surgery or for giant fistulae. Covered stents in general should be avoided due to the high incidence of thrombosis and restenosis, but could be indicated in cases of multiple fistulae.

Conflict of interest: None declared 